\title{
La pédagogie coopérative: De la formation à la mise en pratique
}

\section{Katia Lehraus}

Cet article vise à rendre compte d'une étude portant sur la mise en pratique d'une approche à laquelle des ensei gnants primaires genevois ont été formés: la pédagogie coopérative. Quelques enjeux concernant la formation continue des enseignants sont exposés, ainsi que les aspects méthodologiques de la recherche effectuée. L'analyse des données montre que les planifications d'activités examinées et les pratiques dédarées par les enseignants interviewés correspondent globalement aux caractéristiques de la pédagogie coopérative. Cependant, diverses modalités de mise en oeuvre apparai ssent, liées notamment à la manière de prendre en compte l'aspect social de l'approche. Les éléments de réponse concernant lesfacteurs affectant la mise en pratique permettent de dégager quelques implications pour la formation continueà la pédagogie coopérative.

\section{Probl émat i que}

Les didactiques des disciplines en vigueur dans l'enseignement primaire à $\mathrm{Ge}$ nève préconisent des démarches pédagogiques interactives, s'inscrivant dans une perspective socio-constructiviste et privilégiant notamment le travail en petits groupes. ${ }^{1}$ II ne suffit cependant pas de faire travailler des élèves ensemble pour qu'ils en tirent un bénéfice cognitif individuel (Gilly, 1988). Par ailleurs, les enseignants ${ }^{2}$ se trouvent souvent démunis face aux difficultés de fonctionnement du travail interactif.

Plusieurs courants de recherche ont exploré les aspects de confrontation et de négociation inhérents aux processus sociocognitifs mis en œuvre lors d'interactions sociales entre pairs et leur rôle dans l'apprentissage. Ainsi, les travaux s'inscrivant dans une perspective néopiagétienne ont menéà l'élaboration de la thèse du conflit sociocognitif (D oise \& M ugny, 1997; Perret-Clermont \& N icolet, 1988). D 'autres courants de recherche (Blaye, 1989; Gilly, 1988; Gilly, Fraisse \& Roux, 1988), sans pour autant nier le rôle décisif du conflit sociocognitif, mettent en évidence les diverses modalités de coopération constitutives d'un fonc- 
tionnement interactif favorisant l'apprentissage. Ces études montrent d'un point de vue théorique les bénéfices potentiels de situations interactives. Elles proposent cependant peu de pistes d'ordre pédagogique pour guider les professionnels dans ce type de démarches.

La pédagogie coopérative, telle que définie dans cet article, vise idéalement deux buts: apprendre à coopérer et coopérer pour apprendre. Cette approche cherche d'une part à favoriser une éducation à des valeurs coopératives; d'autre part elle propose des principes, des outils et des stratégies permettant de mieux structurer le travail en équipe afin d'en améliorer l'efficacité et de promouvoir des interactions constructives pour l'apprentissage. D e nombreuses études ( $p$. ex. Slavin, 1990; Johnson \& Johnson, 1989) ont en effet mis en évidence des corré lations positives entre l'utilisation de la coopération et les résultats en apprentissage. II n'est cependant pas évident que cette approche, définie opérationnelle ment par des théoriciens nord-américains, corresponde à la conception de la coopération partagée par les professionnels du vieux continent. Pour les enseignants genevois par exemple, la pédagogie coopérative semble évoquer davantage les courants européens issus des mouvements d'éducation nouvelle, et notamment les idées pédagogiques de Freinet (Lehraus, 1998).

La présente recherche a pour objet d'examiner les effets d'un dispositif de formation continue des enseignants dévolu à la pédagogie coopérative. Plus large ment, l'objet de cette étude de type qual itatif est d'estimer dans quelle mesure des enseignants genevois peuvent sapproprier le corpus théorique d'une approche pédagogique d'origine nord-américaine et l'intégrer à leurs pratiques de classe. Après avoir rappelé les idées essentielles de l'apprentissage coopératif et de la pédagogie coopérative, une seconde section de la partie théorique expose les principes qui ont guidé la formation continue. La suite de l'article est consacrée à la méthodologie de l'étude et, surtout, aux résultats obtenus.

\section{Introduction théorique}

L'apprentissage coopératif et I a pédagogie coopérative Le terme apprentissage coopératif (cooperative learning) désigne un ensemble de méthodes dont l'enjeu est d'organiser une classe en sous-groupes, le plus souvent hétérogènes, au sein desquels les élèves travaillent en coopération sur des tâches scolaires. D iverses approches sinscrivent sous cette appellation générique, dont on peut citer en particulier les méthodes de travail en équipes mises au point par Slavin3 (1990); la méthode du Jigsaw (Aronson, Blaney, Stephan, Sikes \& Snapp, 1978); Learning Together (Johnson, Johnson \& H olubec, 1994); Group Investigation (Sharan, 1994); Structural A pproach (Kagan, 1994); Complex Instruction (Cohen, 1994). Ces méthodes diffèrent notamment par la manière de prendre en compte un certain nombre de principes visant à assurer la coopération au sein des équipes. 
L'apprentissage coopératif repose sur cinq principes de base énoncés par Johnson et al. (1994): une interdépendance positive entre les membres des équipes; une responsabilisati on individuelle et collective face à la tâche; le dével oppement systématique d'habi letés sociales; la promotion d'interactions simultanées en groupes héérogènes restreints, une réflexion critique sur les processus à l'œuvre dans les groupes. Afin d'exemplifier ces principes, considérons une activité de mathématiques destinée à des élèves de sept à huit ans: le «domino des grands nombres»4. En parallèle avec l'objectif disciplinaire (effectuer des additions et des soustractions jusqu'à 100), l'accent est mis sur le développement d'une habileté sociale (respecter le rythme de travail de l'autre). Au sein d'équipes hétérogènes de trois élèves (formées par l'enseignant), l'interdépendance positive est instaurée au moyen d'un but commun (construire ensemble un domino) et d'un partage des ressources (chaque membre reçoit un tiers des pièces). La responsabilisation est assurée par l'attribution de rôles (organisateur, vérificateur, modérateur). Au terme de l'activité, une réflexion critique menée par l'enseignant permet aux élèves d'effectuer un bilan du point de vue des mathématiques et du fonctionnement des équipes.

En même temps qu'elle vise à mettre en œuvre les principes énoncés, la pédagogie coopérative (H owden \& Kopiec, 2000) fait explicitement appel aux valeurs de la coopération ( $p$. ex., la confiance, l'entraide, la solidarité) et cherche ainsi à aller au-delà de la seule utilisation des composantes de l'apprentissage coopératif dans le but d'assurer le bon fonctionnement des équipes de travail. Une pratique de pédagogie coopérative devrait ainsi comporter, outre la structuration des activités selon les cinq principes cités, des moments réservés à d'autres démarches d'éducation à la coopération (p. ex., la résolution de conflits, le conseil de coopération) et viser à développer les valeurs de la coopération. La mise en œuvre d'une telle démarche demande aux praticiens d'examiner leurs conceptions pé dagogiques ainsi que leurs valeurs personnelles et de redéfinir le cas échéant leur propre rôle dans la relation interactive avec les apprenants. L'appropriation et la mise en pratique de la pédagogie coopérative par les professionnels dans leurs contextes respectifs est donc une entreprise ambitieuse.

\section{La formation conti nue des enseignants}

La formation continue des enseignants se trouve actuellement au cœur d'enjeux essentiels, concernant notamment le développement de la professionnalité des praticiens et l'implantation des innovations (Charlier \& Charlier, 1998). O n fait généralement I'hypothèse que les modalités de formation qui auraient le plus d'impact sur un changement de pratiques seraient du type formation-action, formation articulée à la pratique et formation interactive-réflexive (C auterman, $D$ emailly, Suffys \& Bliez-Sullerot, 1999). Cependant, les effets d'une formation dépendent non seulement de facteurs internes, tels les objectifs poursuivis et les stratégies utilisées dans le cadre de la formation (Charlier \& C harlier), mais aussi de facteurs externes, comme la reconnaissance de la hiérarchie (H uberman, 
1989) et un soutien collégial (I shler, Johnson \& Johnson, 1998). D 'autres facteurs encore, internes aux formés ceux-ci, jouent également un rôle important dans l'appropriation et la mise en œuvre de pratiques innovantes. Par exemple, une congruence entre les conceptions pédagogiques des enseignants et l'initiation à des approches concordant avec ces conceptions constitue un facteur favorisant la mise en pratique (Smylie, 1988; Sparks, 1988).

La formation des enseignants à l a pédagogie coopérative La formation des enseignants à la pédagogie coopérative peut s'inspirer directement des recherches effectuées dans le champ de l'apprentissage coopératif, car c'est sous cette étiquette que la grande majorité des chercheurs et praticiens se situent. Les effets des formations sur la mise en pratique à plus long terme de cette approche par les professionnels ont été encore peu étudiés (Ishler et al., 1998). On sait cependant que des enseignants affirmant pratiquer l'apprentissage coopératif n'appliquent pas toujours l'ensemble des principes de base retenus par les chercheurs (Antil, Jenkins, Wayne $\&$ Vadasy, 1998). Par ailleurs, il apparaît que de nombreux professionnels ne persévèrent pas dans la mise en pratique de l'apprentissage coopératif, ou la poursuivent d'une façon peu adéquate qui, au lieu de les mener aux effets bénéfiques escomptés, se termine souvent par des désillusions et des frustrations (Watson, Kenidzor, Dasho, Rutherford \& Solomon, 1998). Selon Roy (1998), les enseignants doivent en effet passer par un certain nombre de stades d'appropriation (H all \& Loucks, 1977), successivement encourageants et décourageants, pour parvenir à une mise en œuvre efficace de l'apprentissage coopératif.

Toujours selon Roy (1998), une formation conçue sur plusieurs années et incluant un soutien collégial ainsi qu'une reconnaissance officielle favorisel'appropriation de l'apprentissage coopératif par les enseignants. D e plus, un coaching organisé au cours des années suivant les séances de formation semble avoir davantage d'impact en termes de mise en pratique que les caractéristiques de la formation elle-même (Johnson \& Johnson, 1998). Enfin, les conceptions des enseignants sur l'importance relative des apprentissages sociaux par rapport aux apprentissages cognitifs constituent un facteur favorisant grandement la mise en oeuvre de l'apprentissage coopératif (Rich, 1990).

\section{Présentation de I a recherche}

Une mise en oeuvre la plus conforme possible aux principes de la pédagogie coopérative et persistant au-delà de la période de formation ne semble donc pas un pari gagné d'avance. Le compte rendu d'une recherche effectuée dans le contexte de l'enseignement primaire genevois se propose d'illustrer cette mise en pratique suite à une formation et d'esquisser quelques pistes de réflexion à ce propos. Les questions de recherche sont organisées sel on deux axes. 
- Un premier faisceau d'interrogations concerne la planification et la mise en pratique de la pédagogie coopérative. Q uelles sont les caractéristiques des activités élaborées par des enseignants pendant la formation suivie? Q uelles sont les pratiques déclarées par quelques enseignants ayant des rapports différents à la pédagogie coopérative?

- Un second faisceau de questionnements touche les facteurs influençant la mise en pratique de cette approche. Q uel est l'impact des conceptions des enseignants? Q uel est l'impact des caractéristiques du contexte et de la formation?

\section{Description du contexte}

Comme indiqué au début de cet article, la pédagogie coopérative, telle que pré sentée dans le cadre de séminai res de formation continue de l'enseignement primaire à G enève, se fonde sur les cinq principes de base de Johnson et al. (1994). Ainsi, cette approche d'inspiration nord-américaine ne correspond pas nécessairement à la conception de la coopération la plus largement répandue au sein du corps enseignant genevois (Lehraus, 1998).

U ne formation continue à la pédagogie coopérative a été proposée, sur inscription personnelle, aux enseignants primaires genevois dans le cadre des offres de perfectionnement sous forme d'un cours d'initiation (six demi-journées ré parties sur l'année scolaire) et d'un séminaire de suivi l'année suivante (quatre demi-journées réparties sur l'année scolaire). La formation était conçue en cohérence avec l'approche pédagogique qui en faisait l'objet, en ce sens que le travail lors des séances était le plus souvent possible organisé en sous-groupes selon les principes de base de la coopération, avec des mises en situation permettant aux enseignants d'expérimenter eux-mêmes le travail coopératif, des travaux de ré flexion en équipes à partir de textes théoriques, des propositions d'activités à expérimenter en classe, des analyses et des échanges à propos de ces expériences, des moments communs de planification d'activités. Après avoir proposé pendant trois années scolai res cette modal ité de formation, il semblait utile de procéder à une prise de données susceptible de dépasser une simple mesure de la satiffaction des participants au terme des sessions de formation.

Mét hodol ogi e 5

Les données pour cette recherche ont été recueillies à travers deux démarches:

- une analyse documentaire des activités de pédagogie coopérative $(n=66)$ élaborées par des enseignants primai res formés à cette approche à G enève entre 1998 et 2001;

- et une analyse de contenu des discours de quelques enseignants $(n=7)$ déclarant pratiquer cette approche, recueillis par entretiens.

Le corpus des activités examinées dans la première phase de la recherche est constitué des documents élaborés, le plus souvent à l'aide d'un support de plani- 
fication, par les enseignants pendant les formations suivies. II sagit del'ensemble exhaustif des activités que leurs auteurs ont bien voulu apporter lors des séances de formation pour un échange avec les collègues et une discussion critique. $\mathrm{Ce}$ corpus a été analysé au moyen d'une grille permettant de mettre en évidence notamment la prise en compte des cinq principes de Johnson et al. (1994).

Les personnes interviewées $(n=7)$ dans la seconde phase de la recherche ont été sélectionnées sur la base d'un questionnaire concis qui a été envoyé à l'ensemble des enseignants ayant suivi la formation $(n=95)$. Elles ont été choisies en fonction des critères suivants: avoir plus de 15 ans d'expérience de l'enseignement; avoir suivi l'ensemble des formations proposées; avoir déclaré une pratique régulière de la pédagogie coopérative; avoir élaboré une ou plusieurs activités du corpus d'analyse. L'option prise visait d'une part à enquêter auprès d'enseignants expérimentés, de façon à éviter une confusion possible entre la variable mise en œuvre d'une nouvelle approche pédagogique et la variable difficultés de gestion de classe fréquemment rencontrées par des praticiens débutants; d'autre part il s'agissait d'accéder à des personnes susceptibles, de par leur intérêt et leur engagement pour la pédagogie coopérative, de présenter des pratiques relativement solides et ancrées, pour saisir en quelque sorte ce qui pouvait être fait «au mieux» dans le contexte en question. Ce choix a été opéré en raison de la nouveauté de la formation proposée, de manière à pouvoir se faire une idée du degré de mise en pratique qu'il est possible d'atteindre. En outre, l'échantillon a été équilibré en tenant compte de l'appartenance des enseignants aux deux cycles ${ }^{6}$ de l'enseignement primaire, pour en apprécier les éventuelles différences. Les raisons invoquées comme source d'intérêt pour la pédagogie coopérative ont également été prises en compte dans l'optique d'accéder à un éventail contrasté de pratiques.

Suite à une première transcription intégrale des entretiens, les propos des enseignants de chaque cycle ont été rassemblés selon un découpage thématique. $\mathrm{Ce}$ corpus a alors fait l'objet d'une analyse de contenu (Bardin, 1977) des discours enregistrés. C elle-ci a été effectuée dans un premier temps par le biais d'une anaIyse qual itative horizontal e centrée sur les thèmes liés aux questions de recherche, en regroupant d'une part les trois enseignants du cycle élémentaire (CE) et d'autre part les quatre enseignants du cycle moyen (CM ). ${ }^{7}$ Certains éléments émergeant selon un axe vertical ont cependant été mis en évidence dans un deuxième temps, d'une part en mettant en parallèle des aspects permettant d'éprouver la cohérence entre les divers thèmes abordés, d'autre part selon une logique intra-individuelle des discours sur des points particuliers, permettant d'accéder à des profils d'enseignants (Blanchet \& Gotman, 1992).

\section{Résul tats}

Comme mentionné plus haut, les interrogations qui ont orienté cette étude concernent d'une part la planification et la mise en pratique de la pédagogie co- 
opérative, d'autre part les facteurs influençant cette mise en pratique. Les résultats sont présentés selon ces deux axes.

La planification et la mi se en pratique de la pédagogie coopérat i ve

\section{Les caractéristiques des activités}

Le tableau 1 présente une vision synthétique des activités de pédagogie coopérative conçues par les praticiens pendant la formation suivie. Celles-ci ont été élaborées à parts égal es par des enseignants du cycle élémentaire (CE) et du cycle moyen (CM ). 8 D u point de vue des disciplines, les activités des deux cycles privilégient le français, puisque plus de la moitié des documents concernent cette branche, alors que les mathématiques ne sont représentées que par un cinquième environ des activités élaborées, un autre cinquième englobant toutes les autres disciplines. Q uant à la taille des groupes, la majorité des activités indiquent des équipes de trois élèves.

Tableau 1: Activités élaborées par l'ensemble des ensei gnants formés à la pédagogie coopérative

\begin{tabular}{|l|c|c|c|}
\hline & $\begin{array}{c}\text { Cycle élémentaire } \\
\text { (CE) }\end{array}$ & $\begin{array}{c}\text { Cycle moyen } \\
\text { (CM) }\end{array}$ & $\begin{array}{c}\text { Total } \\
\text { (CE et CM ) }\end{array}$ \\
\hline $\begin{array}{l}\text { Tendances générales } \\
\text { - N ombre d'activités produites }\end{array}$ & 33 & 33 & 66 \\
- D isciplines concernées: & 18 & 20 & 38 \\
$\quad$ - français & 3 & 10 & 13 \\
$\quad$ - mathématiques & 12 & 3 & 15 \\
- autre & 11 & 1 & 12 \\
- Taille des équipes: & 13 & 21 & 34 \\
$\quad$ - deux élèves & 4 & 10 & 14 \\
$\quad$ - trois élèves & 5 & 1 & 6 \\
$\quad$ - quatre élèves et plus & & & \\
- sans indication & 28 & 33 & 61 \\
\hline Principes de base respectés & 27 & 32 & 59 \\
- Interdépendance positive & 24 & 30 & 54 \\
- Responsabilisation & 21 & 17 & 38 \\
- H abiletés sociales: & 9 & 18 & 27 \\
$\quad$ fonctionnement du groupe & 8 & 8 & 16 \\
$\quad$ - habiletés de communication & 3 & 11 & 14 \\
- valeurs de coopération & 6 & 9 & 15 \\
- recherche de consensus & 24 & 22 & 46 \\
- autre & 16 & 20 & 36 \\
- Réflexion critique & & & \\
- Groupement hétérogène & & & \\
& & & \\
\hline
\end{tabular}


En considérant la traduction des principes de base de la pédagogie coopérative en projets de mise en œuvre dans les planifications des enseignants, il apparaît que la quasi total ité des activités respectent la plupart des cinq principes de Johnson et al. (1994). L'interdépendance positive est instaurée pour 92\% des activités, le plus souvent au moyen d'un but commun, puis d'un partage de ressources suivi d'une répartition des tâches. II est à noter que les deux tiers des activités s'appuient conjointement sur ces trois types d'interdépendance. La responsabilisati on est assurée pour $90 \%$ des activités, le plus souvent à travers l'attribution de rôles. Le développement des habiletés sociales est une composante intégrée à $82 \%$ des activités. La réflexion critique figure dans les planifications de $70 \%$ des activités. Enfin, la décision d'un mode de groupement hétérogène apparaît dans $55 \%$ des activités.

D es différences entre les activités des deux cycles se dessinent au niveau de la taille des groupes et du principe des habiletés sociales. Le nombre d'élèves par équipe est de manière générale plus élevé dans les activités du CM que dans celles du CE. Par ailleurs, les activités du CE privilégient principalement des compé tences utiles au fonctionnement du groupe (p. ex., parler doucement), alors que les activités du CM mentionnent le plus souvent des compétences plus élaborées de communication (p. ex., critiquer positivement).

Ce tableau rapidement brossé des caractéristiques des activités élaborées révèle des documents passablement conformes aux principes de base de la pédagogie coopérative. Les différences entre les deux cycles semblent s'inscrire dans une logique liée à l'âge des élèves. $D$ ans quelle mesure ces planifications d'activités sont-elles représentatives de la réalité des pratiques dans les classes? Les témoignages de sept enseignants, déclarant pratiquer de manière soutenue la pédagogie coopérative depuis environ trois ans, viennent éclairer cette question.

\section{Les pratiques déclarées}

Le tableau 2 présente une vue d'ensemble synthétique de certains aspects des entretiens. L'analyse des discours montre que ces professionnels possèdent des conceptions théoriques relativement élaborées à propos de la pédagogie coopérative. En effet, les cinq principes de base de l'approche sont mentionnés par la majorité des enseignants du cycle moyen (CM ) et quatre des cinq principes par l'ensemble des enseignants du cycle élémentaire $(C E)$. D ans les définitions de l'approche données par les trois enseignants $C E$, l'accent est mis sur le dével oppement des habiletés sociales en parallèle avec les apprentissages scolaires: «c'est diviser une tâche en habiletés social es et sur la tâche, voir bien ces deux niveaux» (CE1). En contraste, les définitions des quatre enseignants CM sont en majorité orientées vers les aspects organisationnels du travail d'équipe: «c'est une façon d'organiser le travail au niveau des élèves pour qu'ils réalisent une activité ensemble, pour atteindre un même but et avec une structure établie... c'est apprendre à travailler en groupe» (CM 4). Par ailleurs, deux enseignants CM considèrent les cinq principes comme solidaires: «si on laisse de côté une des 
composantes, ce n'est plus de la pédagogie coopérative... c'est un tout, il y a un certain nombre de critères» (CM 1).

Tableau 2: Pratiques déclarées par les ensei gnants intervi ewés

\begin{tabular}{|c|c|c|c|}
\hline & $\begin{array}{l}\text { Cycle élémentaire } \\
\text { (CE: } 3 \text { enseignants) }\end{array}$ & $\begin{array}{l}\text { Cycle moyen } \\
\text { (CM : } 4 \text { ensei- } \\
\text { gnants) }\end{array}$ & $\begin{array}{c}\text { Total } \\
\text { (CE et CM) }\end{array}$ \\
\hline $\begin{array}{l}\text { Conceptions théoriques } \\
\text { - Principes de base: } \\
\text { - interdépendance positive } \\
\text { - responsabilisation } \\
\text { - habiletés sociales } \\
\text { - groupement hétérogène } \\
\text { - réflexion critique } \\
\text { - autre }\end{array}$ & $\begin{array}{c}3 \\
3 \\
3 \\
1 \\
3 \\
\text { objectif cognitif et } \\
\text { social (2) }\end{array}$ & $\begin{array}{c}3 \\
4 \\
4 \\
3 \\
4 \\
5 \text { principes soli- } \\
\text { daires (2) }\end{array}$ & $\begin{array}{l}6 \\
7 \\
7 \\
4 \\
7\end{array}$ \\
\hline $\begin{array}{l}\text { D escription des pratiques } \\
\text { - Eléments soulignés } \\
\text { - D isciplines pratiquées: } \\
\text { - français } \\
\text { - mathématiques } \\
\text { - autre } \\
\text { - Types d'activités } \\
\text { - Aspects privilégiés } \\
\text { - Eléments menant au succès } \\
\text { - Eléments posant problème } \\
\text { - Effets observés }\end{array}$ & $\begin{array}{c}\text { planification (3) } \\
3 \\
3 \\
2 \\
\text { climat de classe (2) } \\
\text { habiletés sociales (3) } \\
\text { structure(3), contenu(2) } \\
\text { âge élèves (2) } \\
\text { positifs (3) }\end{array}$ & $\begin{array}{c}\text { planification (4) } \\
4 \\
4 \\
2 \\
\text { critèreschoix tâche(4) } \\
\text { planif.(2), cognitif(1), } \\
\text { social(1) } \\
\text { structure (4) } \\
\text { gestion (3) } \\
\text { positifs(3), doutes(3) }\end{array}$ & $\begin{array}{l}7 \\
7 \\
4\end{array}$ \\
\hline $\begin{array}{l}\text { Intérêts pour l'approche } \\
\text { - Sources de l'intérêt } \\
\text { - Raisons de l'intérêt } \\
\text { - Réponses apportées }\end{array}$ & $\begin{array}{l}\text { intérêt personnel (3) } \\
\text { valeurs(2), interactions(2) } \\
\text { rejoint valeurs per- } \\
\text { sonnelles (3) }\end{array}$ & $\begin{array}{l}\text { pratique insatisfai- } \\
\text { sante (4) } \\
\text { efficacité(3), cognitif(2) } \\
\text { aide gestion groupes } \\
\text { (3) }\end{array}$ & \\
\hline
\end{tabular}

Au niveau de la description des pratiques, on observe pour l'ensemble des professionnels un souci de choisir une tâche adéquate pour être organi sée sous forme coopérative, en articulation, si possible, avec les objectifs d'apprentissage. Plusieurs enseignants signalent des pratiques comportant des facettes structurées, planifiées à l'avance, et des manières plus informelles de mettre les élèves en coopération: «une fois par jour, d'une manière ou d'une autre, on coopère [...] cela peut être vite fait, par deux [... et une fois par semaine, j'essaie de faire une activité plus structurée» (CM 2). O n constate que certains enseignants se sont ainsi appropriés l'état d'esprit de la pédagogie coopérative et sont conscients d'une évolution dans leurs pratiques: «au départ, on se dit, je veux faire une activité de pédagogie coopérative, qu'est-ce que je fais? et maintenant, c'est je veux faire du français, tiens je vais le faire sous cette forme-là» (CM 1). 
Les disciplines pratiquées en pédagogie coopérative sont surtout, et pour l'ensemble des personnes, le français et les mathématiques. Pour les autres branches, la majorité des enseignants CE utilisent cette approche dans divers domaines, alors que la plupart des enseignants CM semblent davantage restreindre leurs pratiques aux deux disciplines dites principales.

Du point de vue des types d'activités proposés en pédagogie coopérative, la majorité des enseignants CE mettent la priorité sur l'instauration d'un climat de classe avant d'entrer dans des contenus d'apprentissage. Les enseignants CM insistent quant à eux davantage sur les critères de choix pour décider de planifier une tâche en pédagogie coopérative, ces critères étant très différents voire contradictoires d'une personne à l'autre. II est à noter cependant que les enseignants CM ne mentionnent à aucun moment le climat de classe, perçu comme prioritaire par leurs collègues du CE. D'autres éléments viennent renforcer ces constats. En interrogeant les professionnels sur les aspects de la pédagogie coopérative qu'ils privilégient, les enseignants CE mettent clairement l'accent sur le travail des habiletés sociales: «je travaille avec le tableau en $T^{9}$ [...] c'est très souvent l'aspect social qui me guide» (CE3). Chez les enseignants CM en revanche, les éléments privilégiés sont moins homogènes. Une personne souligne égale ment l'aspect social, deux enseignants mettent l'accent sur la prise en compte des principes de base lors de la planification, une personne enfin favorise l'aspect cognitif des activités.

En évoquant les éléments qui aident au bon fonctionnement des activités de coopération en classe, c'est globalement la mise en œuvre des aspects structurés del'approche qui est perçue par l'ensemble des enseignants comme le facteur essentiel de réussite. Le principe de responsabilisation, bien que conçu de diverses manières selon les personnes, est fréquemment cité par les professionnels des deux cycles: «je leur dis qu'à la fin je peux poser des questions à tout un chacun... $c^{\prime}$ est aussi un bon moyen de contrôle» (CM 4). Plusieurs personnes relèvent en outre l'intérêt pour les élèves de bénéficier ainsi d'un cadre rassurant. Par ailleurs, certains éléments sont considérés comme problématiques lors des activités en coopération. Les enseignants CE invoquent généralement l'âge des élèves comme source de difficultés: «se décentrer, c'était ce qui était difficile pour les petits» (CE1). Les enseignants CM quant à eux soulignent surtout les difficultés de gestion en cours d'activité ou des problèmes de fonctionnement voire de discipline: «je n'arrive plus à voir comment intervenir, j'ai l'impression de papillonner, de ne pas être assez efficace en tout cas au niveau apprentissage [...] il y a beaucoup de choses à gérer» (CM 2).

Lorsqu'on se penche sur les sources de l'intérêt pour la pédagogie coopérative, on constate que pour les enseignants CE, l'approche correspond avant tout à un intérêt personnel: «cela colle tout à fait à mes valeurs en tant que personne... les activités de coopération sont un bon vecteur pour transmettre ces valeurs» (CE3). Pour les enseignants CM en revanche, il s'agit en premier lieu de trouver des pistes face à une pratique de travail de groupe insatisfaisante. Lors d'une par- 
tie structurée de l'entretien, il était demandé aux enseignants de choisir entre plusieurs rai sons possibles de s'intéresser à la pédagogie coopérative. Les choix effectués viennent renforcer ces constats. La plupart des enseignants CE mettent en effet la priorité sur le développement des valeurs de coopération puis sur la qualité des interactions entre élèves; la majorité des enseignants CM sont intéressés d'abord par l'efficacité du travail de groupe puis par les effets sur l'apprentissage grâce aux interactions. D e manière cohérente, les réponses apportées par la pédagogie coopérative apparaissent au niveau de l'aide à la gestion du travail de groupe chez la plupart des enseignants CM : «'ai trouvé quelque chose qui cadre la recherche par groupes [...] làj'ai l'impression qu'ils sont vraiment au travail» (CM 2).

Afin d'éprouver la cohérence entre les conceptions théoriques et les pratiques déclarées par les enseignants, les éléments de discours liés à la mise en oeuvre de la pédagogie coopérative ont été examinés, pour y déceler l'actualisation des principes de base ou leur éventuel manque. $0 \mathrm{n}$ peut constater ainsi que le principe du groupement hétérogène, implicitement absent au niveau des conceptions théoriques des enseignants CE, apparait clairement dans l'explicitation des pratiques de ces trois personnes. $C$ hez les enseignants $C M$, en revanche, le principe de dével oppement des habiletés sociales, pourtant annoncé comme fai sant partie de l'approche, n'est réellement mis en œuvre que par la moitié des personnes, mettant en évidence un décal age explicite sur le plan des pratiques.

Ce bref examen des pratiques déclarées par les enseignants montre qu'elles se révèlent, comme les activités planifiées, relativement cohérentes par rapport aux principes de base de la pédagogie coopérative. La distinction majeure entre les deux cycles se situe au niveau de l'importance accordée au développement des habiletés social es. $D$ ans quelle mesure cette image des pratiques est-elle influencée par des facteurs internes et externes aux formés?

\footnotetext{
Les facteurs influençant la mi se en pratique de la pédagogie coopérative
}

\section{Les conceptions des enseignants}

La quasi totalité des professionnels mentionnent l'intérêt des interactions entre pairs. Toutefois on perçoit, chez la plupart des enseignants $C M$, des difficultés à concevoir ou assumer le statut différent que cela implique pour l'enseignant: «accepter de laisser la responsabilité aux enfants, cela demande une remise en question terrible [...] c'est l'anti-thèse de ce qu'est un enseignant [...] accepter de cé der une part de son autorité ce n'est pas évident et il y a tout un chemin à faire dans sa tête» (CM 1). Q uant aux enseignants $C E$, tous affirment que la pédagogie coopérative correspond à leurs valeurs personnelles et que la mise en pratique de l'approche leur permet ainsi de les transmettre: «c'est un idéal de société qui m'habite et que je trouve génial de pouvoir promouvoir» (CE1).

Pour nuancer quelque peu l'image proposée jusqu'à présent, séparant les enseignants par cycle au risque de figer une dichotomie entre ces deux catégories de 
professionnel ${ }^{10}$, considérons à présent les profils présentés dans le tableau 3. La répartition en trois catégories est bien sûr schématique, mais elle prend en compte les grandes tendances qui se dégagent des propos de chaque enseignant.

Les personnes du profil I montrent un intérêt personnel pour la pédagogie coopérative, lié à leurs propres val eurs en tant qu'individus et aussi parce qu'elles y voient un avantage au niveau du développement d'interactions de qualité entre élèves. L'approche leur permet ainsi de transmettre ces valeurs qui ont de l'importance à leurs yeux, en revanche les bénéfices escomptés pour l'apprentissage ne se dégagent pas explicitement de leurs propos. Au niveau des pratiques, on voit que ces enseignants privilégient les habiletés sociales et qu'ils observent des effets positifs à ce niveau, en même temps qu'ils relèvent l'importance de la structuration des activités comme facteur de succès des démarches proposées. Leur mise en œuvre de l'approche paraît satisfai sante et cohérente avec leurs conceptions; les principaux problèmes qu'ils rencontrent sont attribués à l'âge des élèves.

Tableau 3: Profils des enseignants interviewés

\begin{tabular}{|c|c|c|c|}
\hline & Profil I (2 CE) & $\begin{array}{c}\text { Profil II (1 CE, } 2 \\
\text { CM) }\end{array}$ & Profil III (2 CM ) \\
\hline $\begin{array}{l}\text { Conceptions des personnes } \\
\text { - Conceptions pédagogiques } \\
\text { - Conceptions personnelles }\end{array}$ & $\begin{array}{l}\text { - interactions } \\
\text { - valeurs decoopéra } \\
\text { tion }\end{array}$ & $\begin{array}{l}\text { - interactions, statut } \\
\text { maître } \\
\text { - valeurs de coopéra } \\
\text { tion }\end{array}$ & $\begin{array}{c}\text { - interactions } \\
-\end{array}$ \\
\hline $\begin{array}{l}\text { Intérêt pour approche } \\
\text { - Sources de l'intérêt } \\
\text { - Raisons de l'intérêt } \\
\text { - Réponses apportées }\end{array}$ & $\begin{array}{l}\text { - intérêt personnel } \\
\text { - valeurs de coopéra } \\
\text { tion et qualité in } \\
\text { teractions } \\
\text { - valeurs person } \\
\text { nelles }\end{array}$ & $\begin{array}{l}\text { - pratique insatisfai } \\
\text { sante } \\
\text { - efficacité travail de } \\
\text { groupe et valeurs } \\
\text { de coopération } \\
\text { - gestion et valeurs } \\
\text { personelles }\end{array}$ & $\begin{array}{l}\text { - pratique insatisfai } \\
\text { sante } \\
\text { - efficacité travail de } \\
\text { groupe et bénéfices } \\
\text { cognitifs } \\
\text { - }\end{array}$ \\
\hline $\begin{array}{l}\text { D escription des pratiques } \\
\text { - Principes de base manquants } \\
\text { - Aspects privilégiés } \\
\text { - Eléments induisant le succès } \\
\text { - Eléments posant problème } \\
\text { - Effets observés }\end{array}$ & $\begin{array}{l}\text { - habiletés sociales } \\
\text { - structure } \\
\text { - âge élèves } \\
\text { - constats positifs }\end{array}$ & $\begin{array}{l}\text { - habiletés sociales } \\
\text { - structure et } \\
\text { contenu } \\
\text { - constats positifs }\end{array}$ & $\begin{array}{l}\text { - habiletés sociales } \\
\text { - planification, co } \\
\text { gnitif } \\
\text { - structure } \\
\\
\text { - gestion } \\
\text { - doutes }\end{array}$ \\
\hline
\end{tabular}

A l'autre extrémité, les enseignants du profil III ne révèlent pas une adhésion explicite aux valeurs de la coopération et la pédagogie coopérative les intéresse en tant que piste possible face à une pratique de travail de groupe insatisfaisante. 
Leurs attentes se situent ainsi en priorité au niveau de l'efficacité du travail de groupe et des bénéfices cognitifs qui y seraient associés. Les aspects privilégiés par les personnes appartenant à ce profil sont la planification et les aspects cognitifs; en revanche, ils ne mettent pas en pratique le principe de développement des habiletés sociales. La mise en œuvre semble fonctionner grâce aux éléments structurels de l'approche. C es enseignants rencontrent néanmoins des problèmes de gestion et sont partagés sur l'efficacité de leurs démarches.

Enfin, les enseignants du profil II se situent dans un entre-deux incluant des éléments de chacun des deux profils décrits ci-dessus. II peut donc être défini comme un profil mixte, penchant plus ou moins vers l'un ou l'autre des pôles seIon les circonstances, mais dont la particularité réside dans le fait que ces personnes sont concernées à la fois par les aspects liés aux habiletés sociales et aux valeurs de la coopération et par ceux liés à l'efficacité du travail de groupe à des fins d'apprentissage.

\section{Les caractéristiques du contexte et de la formation}

Les enseignants des deux cycles s'accordent globalement sur les facteurs favorisant ou freinant leur mise en pratique de la pédagogie coopérative. D ans l'ensemble, on peut noter que les constats proposés par les enseignants CM mettent en évidence plus d'aspects négatifs que ceux des enseignants CE. Le tableau 4 présente une vision synthétique des éléments du contexte scolaire et de la formation susceptibles de jouer un rôle au niveau de la mise en pratique de la pédagogie coopérative.

Tableau 4: Facteurs perçus comme favorisant ou freinant la mise en pratique de la pédagogie coopérative

\begin{tabular}{|c|c|c|}
\hline & $\begin{array}{l}\text { Facteurs perçus comme favorisant la } \\
\text { mise en pratique }\end{array}$ & $\begin{array}{l}\text { Facteurs perçus comme } \\
\text { freinant la mise en pratique }\end{array}$ \\
\hline $\begin{array}{l}\text { Contexte } \\
\text { scolaire }\end{array}$ & $\begin{array}{l}\text { Suivi volée (5), horaire souple (5) } \\
\text { M oyens d'enseignement en mathéma- } \\
\text { tiques (4) } \\
\text { M ilieu socioculturel défavorisé (1) } \\
\text { Réseau et échanges entre enseignants (5) } \\
\text { Constats positifs (6) }\end{array}$ & $\begin{array}{l}\text { Effectifs élevés (3), grille horaire morcelée (2) } \\
\text { M anque de moyens didactiques adéquats } \\
\qquad(3) \\
\text { M ilieu socioculturel privilégié (1) } \\
\text { M anque de soutien institutionnel (2) } \\
\text { D ifficultés constatées, doutes (3) }\end{array}$ \\
\hline $\begin{array}{l}\text { Formation } \\
\text { à la } \\
\text { pédagogie } \\
\text { coopéra- } \\
\text { tive }\end{array}$ & $\begin{array}{l}\text { Perception positive de la formation (6): } \\
\text { - alternance nécessaire entre théorie et pratique } \\
\text { - importance des aspects interactifs et réflexifs } \\
\text { - utilité des documents théoriques et pratiques } \\
\text { Public cible (2): } \\
\text { - enseignants expérimentés ayant éprouvé } \\
\text { les limites du travail de groupe } \\
\text { - conviction personnelle ou pédagogique forte }\end{array}$ & $\begin{array}{l}\text { M anque de formation supplémentaire } \\
\qquad \text { pour poursuivre (2) } \\
\text { Difficulté, lourdeur de l'approche (7): } \\
\text { - préparation (conception, matériel... ) } \\
\text { - gestion (mise en route, interventions... ) }\end{array}$ \\
\hline
\end{tabular}


0 utre des plages de temps suffisamment longues à disposition dans la grille-horaire, les constats effectués par les professionnels montrent que le suivi d'une volée sur deux ans permet aux élèves d'intégrer beaucoup de choses:

j'ai vraiment ce gen re de résultats, des enfants qui sentraident, qui sécoutent... et avec une nouvelle volée, il faut reconstruire (CE3);

maintenant ils savent sorganiser [...] les choses viennent relativement naturellement [... ] il y a moins de règles de ma part (CM 1);

je viens de donner mesélèves à ma collègue [...] et ilsont tout de suite dit, bon qui prend quel rôle (CM 3).

C es illustrations mettent en évidence les effets plus spécifiques soulignés par des enseignants appartenant aux trois profils définis plus haut. D ans le premier cas (CE3, profil I), les effets relevés se situent surtout dans le domaine des habiletés sociales. D ans le second cas (CM 1, profil II), c'est I'aspect organisationnel qui se dégage dans une perspective d'efficacité pour le travail scolaire. Enfin, dans le troisième cas (CM 3, profil III), les élèves semblent avoir intégré surtout un aspect structurel relativement superficiel (les rôles).

Les caractéristiques socioculturelles du quartier sont peu évoquées, de même que l'hétérogénéité de la classe. D eux enseignants mettent en évidence un décalage entre les valeurs des familles et celles qu'ils pourraient promouvoir à travers la pé dagogie coopérative. II apparaît moins aisé d'affirmer les valeurs de l'école dans un contexte privilégié, où les valeurs desfamilles semblent avoir plus de poids et de lé gitimité, l'enseignant concerné se montrant réticent à travailler les habiletés sociales: «c'est un milieu élitiste, alors l'aspect social, je n'insiste pas trop [...] je doute que la coopération soit perçue comme un enrichissement personnel» (CM 3).

L'échange entre adultes semble favoriser la mise en pratique de la pédagogie coopérative. Si certains enseignants CM évoquent l'apport d'une collaboration entre collègues, il s'agit de personnes qui, de par leur contexte (d'autres enseignants de l'école sont formés) ou de par leur fonctionnement (duo pédagogique), sont amenées à le faire facilement. Les enseignants C E mettent quant à eux l'accent sur l'importance des échanges au sein d'un réseau informel interécoles qu'ils ont constitué: «c'est grâce à cela que je n'oublie pas la pédagogie coopérative [...] ce groupe est une forte motivation» (CE2). Le réseau semble perçu par les enseignants CE comme une manière de poursuivre une mise en pratique «formatrice»: «e groupe recentre, du point de vue théorique et du point de vue des activités [...] il y a eu les cours et il y a l'après-cours, qui est le réseau, et cela forme un tout, c'est une suite» (CE3).

Pour la quasi totalité des enseignants, la formation reçue est perçue de manière positive et un manque d'offre de suivi est relevé. La pédagogie coopérative est cependant considérée comme une approche exigeante par l'ensemble des personnes interviewées: «on n'est pas au repos en tant que prof [...] il y a le côté astreignant, formel... la pédagogie coopérative est un peu lourde» (CM 3). D eux personnes précisent que c'est une approche utile pour des enseignants expérimentés, qui ont atteint les limites du travail de groupe habituel: 
j'imagine mal quelqu'un qui démarre dans ce métier se lancer tout de suite dans la pédagogie de coopération, cela demande une grande maîtrise des programmes... pas mal d'expérience et je crois qu'il faut avoir eu des échecs, des frustrations, pour se dire que ce qu'on obtient par la pédagogi e de coopération, c'est tellement confortable, quel que soit le prix à payer au départ (CE3).

\section{Discussion}

Synthèse des résult ats

L'apparente fidélité des activités élaborées aux principes de base de la pédagogie coopérative est sans doute influencée par la grille de planification, utilisée par la plupart des enseignants, et dont les rubriques incitent à définir l'actual isation de chaque principe. Par ailleurs, le corpus examiné est constitué de traces écrites de planifications qui d'une part sont destinées à être partagées avec d'autres collègues en séance de formation et susceptibles ainsi d'être soumises à critique, et qui d'autre part représentent des projets de mise en œuvre dont la réalisation ne correspond pas nécessairement aux intentions d'enseignants encore novices en la matière. Le groupement hétérogène, moins apparent que les autres principes dans les activités planifiées, serait-il perçu plutôt comme un aspect plus personnel et adaptable, car davantage lié au contexte et au type de tâche, qu'en tant que principe à respecter au même titre que les autres?

Si les conceptions théoriques des enseignants interviewés dénotent une définition très exigeante de la pédagogie coopérative, celle-ci ne correspond pas pour tous à une transposition fidèle dans la pratique, rejoignant d'autres constats montrant qu'un grand nombre d'enseignants n'utilisent pas l'ensemble des cinq principes de base (Antil et al., 1998). S'agit-il d'une question d'appropriation ou de transformation de l'approche? 0 n pourrait en effet penser que les personnes soulignant la nécessité de prendre en compte tous les principes de base se trouvent à un niveau d'appropriation proche du stade de mechanical use, au sens de $\mathrm{H}$ all et Loucks (1977), durant lequel elles sont engagées dans une recherche de maitrise des éléments de l'approche pour être en mesure de la mettre en pratique. Cependant, certains enseignants manifestent un niveau d'appropriation de l'ordre du stade de refinement, en faisant preuve d'un usage varié de la démarche et en l'adaptant en fonction d'effets escomptés à court terme ou à plus long terme (H all \& Loucks).

$D$ ans les pratiques déclarées, la différence majeure entre les deux cycles se situe au niveau des habiletés sociales, privilégiées par les enseignants $C E$ et n'étant de fait pratiquées que par la moitié des enseignants $C M$. Faut-il considérer la mise à l'écart d'un des principes comme un manque par rapport aux caractéristiques de l'approche, ou comme une transformation, au sens d'Antil et al. (1998), liée aux conceptions personnelles ou au contexte scolaire des professionnels? D ans quelle mesure peut-on se passer du principe de développement des 
habiletés sociales? Les enseignants $C E$ se montrent en effet très attentifs aux relations social es dans la classe, alors que les enseignants CM le sont davantage à la structuration des activités en vue de bénéfices cognitifs, au risque d'occulter la facette sociale de l'approche. Le fonctionnement insatisfaisant mis en évidence par la plupart de ces derniers serait-il lié à un manque de développement systématique des habiletés sociales ou indiquerait-il un regard critique sur leurs pratiques, présageant une évolution future?

Un lien apparaît entre les raisons de l'intérêt des enseignants pour la pédagogie coopérative et l'adéquation des réponses qu'elle apporte à leurs préoccupations, car chacun semble y trouver des pistes de solution aux aspects qui lui tiennent plus particulièrement à cœur. Cependant cette approche, proposée à I'origine par ses concepteurs comme une réponse possible aux problèmes liés aux différences interethniques, à l'hétérogénéité des populations et aux difficultés de gestion de classes nombreuses (Abrami, Chambers, Poulsen, D e Simone, D 'Appolonia \& H owden, 1996), ne parait pas considérée en tant que piste de solution à ces problèmes précis, puisque le contexte est relativement peu évoqué par les enseignants. Par ailleurs, le manque de reconnaissance institutionnelle déploré par deux personnes semble perçu comme une absence de légitimité pour la mise en pratique quotidienne d'une approche ne privilégiant pas uniquement les aspects cognitifs. Si un encadrement supplémentaire semble souhaitable pour compléter la formation, les trois enseignants $C E$ ont comblé ce manque en créant un réseau inter-écoles. Ces constats rejoignent ceux d'I shler et al. (1998) montrant que le degré d'utilisation de l'apprentissage coopératif est lié au degré de support psychologique reçu et à l'appartenance à un réseau de collègues expé rimentant cette approche.

\section{El éments de réponse}

En nous référant aux profils esquissés plus haut, une distinction semble s'établir entre les personnes en fonction du degré de prise en compte de l'aspect social de l'approche coopérative, menant à des modalités de mise en œuvre différentes. O n observe ainsi des pratiques mettant un accent fort sur le développement des valeurs de coopération et des habiletés sociales (profil I), dont une dérive possible serait une survalorisation de l'aspect social au détriment de l'apprentissage cognitif, considéré comme un effet collatéral. Par ailleurs, d'autres pratiques apparaissent, intégrant de manière mesurée un travail sur les habiletés sociales (profil II), mais dont une dérive possi ble serait une survalorisation des aspects organisationnels laissant peu de place aux valeurs de la coopération. Enfin, on observe des pratiques ne prenant pas en compte les habiletés sociales (profil III) et comportant quant à elles le risque de décevoir par le peu d'effets constatés en regard de l'efficacité escomptée au niveau de la gestion et des apprentissages.

Le point clé différenciant ces modalités semble donc lié au développement des habiletés et des valeurs de coopération. L'accent mis sur l'aspect social est-il inversement proportionnel à celui placé sur l'aspect cognitif? En s'engageant 
dans une approche coopérative, chaque professionnel est en effet amené à s'interroger sur la place qu'il souhaite ou qu'il peut, de par son mandat, accorder aux apprentissages sociaux par rapport aux apprentissages cognitifs:

cela me donne des outils pour faire quelque chose dans des valeurs qui me sont chères... je me dis que l'école doit être lelieu où on apprend ce genre de choses [...] on ne sest pas choisi, on vit ensemble, il faut faire quelque chose pour qu'on sentende [...] mais mon mandat par rapport à l'institution, c'est quoi? (CM 2).

Si l'aspect social est écarté, peut-on réellement parler de démarches de pédagogie ou d'apprentissage coopératif? Suffit-il d'injecter de l'interdépendance positive et de la responsabilisation dans des situations de travail de groupe et de prévoir une réflexion critique pour sinscrire sous cette appellation? Le développement des habiletés sociales en parallèle avec les apprentissages cognitifs nous paraît être un critère pertinent permettant de distinguer une approche coopérative d'un travail de groupe habituel, même enrichi de quelques structures supplémentaires.

$D$ ans quelle mesure un dispositif de formation peut-il se révéler efficace sil ne s'inscrit pas en même temps dans une cohérence forte avec les conceptions des formés? Les praticiens qui utilisent la pédagogie coopérative sont essentiellement ceux dont les conceptions pédagogiques et/ou personnelles se trouvent déjà en cohérence générale avec cette approche. C es constats rejoignent les affirmations de Rich (1990), qui met en évidence les relations entre les croyances des enseignants et la qualité de la mise en œuvre de méthodes d'enseignement en rapport avec ces dernières, en soulignant la nécessité de prendre en compte non seulement les conceptions pédagogiques, mais aussi celles qui relèvent davantage de la sphère idéologique. La préoccupation à propos du «statut» de l'enseignant révélée par les propos de la plupart des enseignants $\mathrm{CM}$ pourrait-elle par exemple gé nérer des difficultés dans la délégation aux élèves d'une partie de l'autorité et de la responsabilité dans les apprentissages?

La modalité adoptée pour la mise en pratique de la pédagogie coopérative, en l'occurrencel'accent mis sur l'aspect cognitif et/ou social, semble surtout liée aux conceptions personnelles. $\mathrm{C}$ e constat rejoint le postulat de $\mathrm{C}$ harlier et $\mathrm{C}$ harlier (1998) concernant la relation entre «savoirs enseignants» et «savoirs sur ou pour l'enseignement» (Raymond, 1993), les premiers étant des connaissances «contextualisées, spéculatives et instrumentales» (Clark \& Lampert, 1986) construites par les enseignants au cours de leurs pratiques, les seconds étant constitués en de hors de la pratique pédagogique. Selon $\mathrm{C}$ harlier et Charlier, les «savoirs enseignants» d'un professionnel serviraient de fondements pour éval uer la pertinence de «savoirs sur ou pour l'enseignement», en vue de leur éventuelle intégration à ses pratiques habituelles. Vu l'importance des conceptions personnelles des enseignants quant à leur sensibilité pour la pédagogie coopérative, on pourrait se demander dans quelle mesure cette approche peut intéresser des personnes qui valoriseraient davantage la compétition et/ou dont le modèle pédagogique serait plutôt de type transmissif. La forte congruence observée entre les conceptions personnelles des enseignants et les enjeux de la pédagogie coopérative engage 
ainsi un débat sur la formation des enseignants à cette approche et plus particulièrement sur les moyens d'y rendre réceptives des personnes ayant des conceptions éloignées des pratiques visées.

Plusieurs recherches ont mis en évidence un «problème de transfert» entre la formation et le contexte d'application. Parmi les facteurs susceptibles d'avoir des effets sur ce transfert, on trouve notamment les caractéristiques des formés, la stratégie de formation et l'environnement de travail (Baldwin \& Ford, 1988). Comme le soulignent Johnson et Johnson (1998), I'appartenance à un réseau d'enseignants à la suite de la formation constitue un facteur essentiel à la mise en œuvre sur le long terme. Cette condition, dont bénéficient les trois enseignants CE de par leur propre initiative, montre des personnes très concernées par cette approche pédagogique et qui trouvent dans ce réseau une légitimité, une motivation pour poursuivre ainsi qu'un moyen de régulation de leurs pratiques. O n peut imaginer cependant que la majeure partie des enseignants formés se trouvent très éloignés de ce profil. L'aspect lié aux conceptions des enseignants demeure ainsi une vraie question, car il semble peu probable que la formation seule soit en mesure de susciter des changements chez des personnes idéologiquement éloignées de ce type d'approche et satisfaites de leurs pratiques.

\section{Conclusion}

$\mathrm{N}$ otre recherche menée auprès d'enseignants genevois ne dévoile que certaines facettes de la mise en pratique de la pédagogie coopérative et comporte aussi des limites méthodologiques. Si le choix d'interviewer des personnes très impliquées dans l'approche a permis de mettre en évidence diverses modalités de mise en pratique, il n'autorise pas une réelle estimation de l'impact de la formation sur l'ensemble des participants. D es données Iongitudinales serai ent en outre nécessaires pour pouvoir apprécier la progression des enseignants et des élèves dans l'appropriation de l'approche. Par ailleurs, des observations en classe offriraient l'opportunité de capter ces réalités de manière plus directe. Enfin, il serait important d'affiner l'examen des planifications d'activités à la lumière des didactiques des disciplines pour évaluer le bien-fondé des modifications et adjonctions apportées aux activités issues des moyens d'enseignement officiels.

Les résultats présentés ici permettent néanmoins d'esquisser quelques implications pour la formation continue. Si la pédagogie coopérative reste une approche dont l'usage est laisséà l'appréciation des professionnels, il semble peu judicieux de vouloir susciter l'adhésion de personnes peu concernés par ce type de démarches. Pour les enseignants intéressés en revanche, et dans la perspective d'une mise en pratique à long terme, la formation devrait être conçue sur plus de deux ans, viser en prioritéla constitution d'un réseau et prévoir un suivi au terme des sessions sous une forme qui reste à définir ( $p$. ex. des permanences, des observations en classe, des démarches de coaching). Un tel dispositif permettrait d'accompagner les professionnels dans leur progression à travers les divers stades d'appropriation menant à une pratique intégrée et satisfai sante, autant pour les 
enseignants que pour leurs élèves, comme par exemple celle esquissée à travers les propos suivants:

la pédagogie de coopération me permet de rassembler toutes mes valeurs et mes pratiques [...] j'ai et le travail de groupe, et le partage, et l'intérêt des enfants, et un climat de classe super agréable, et des enfantsqui sont actifs [... ] pour moi c'est le rêve, honnêtement (CE3).

Notes

1 Selon le dossier Les objectifs d'apprentissage de l'école primaire genevoise (D irection de l'enseignement primaire, 2000).

2 Lemasculin a été retenu pour des rai sons de lisibilité, mais ce terme inclut les enseignantes et les enseignants.

3 II s'agit des méthodes STAD (Student Teams-Achievement Divsions), TGT (Teams-GamesTournaments), TAI (Team Assisted Individualization) et CIRC (Cooperative Integrated Reading and Composition) (Slavin, 1990).

4 Activité créée par une enseignante primaire genevoise.

5 C et article est basé sur un mémoire de diplôme d'études approfondies en sciences del'éducation, dont une copie (incluant des annexes qui comportent des précisions méthodologiques) est déposée à la bibliothèque de la faculté de psychologie et des sciences de l'éducation de l'université de $G$ enève.

6 Pour désigner les divisions élémentaire et moyenne de l'enseignement primaire genevois, les termes de cycle élémentaire et cycle moyen sont actuellement utilisés dans la plupart des documents officiels.

7 L'essai-pilote mené avec un enseignant CM n'ayant pas conduit à modifier la trame d'entretien, celui-là a été exploité au même titre que les six autres entretiens prévus ( 3 CE et 3 CM).

8 Le cycle élémentaire regroupe les degrés de lère et 2ème enfantine ainsi que de lère et 2ème primaire (élèves de 4 à 8 ans), alors que le cycle moyen s'étend de la 3ème primaire à la 6ème primaire (élèves de 9 à 12 ans).

9 Le «tableau en T » est une des manières proposées par les approches de pédagogie coopérative pour envisager un travail systématique sur les habiletés sociales, voir p. ex. Abrami et al. (1996).

10 Soulignons le caractère essentiellement exploratoire des analyses présentées ici, eu égard au nombre restreint de sujets ( $\mathrm{N}=7$ ). La comparaison CE versus CM doit, en particulier, être considérée avec prudence puisqu'elle oppose trois et quatre enseignants.

Réf érences bi bl i ographi ques

Abrami, P.C., Chambers, B., Poulsen, C., De Simone, C., D 'Appolonia, S. \& H owden, J. (1996). L'apprentissage coopératif: Théories, méthodes, activités. M ontréal: Editions La Che nelière.

Antil, L.R., Jenkins, J.R., Wayne, S.K. \& Vadasy, P.F. (1998). Cooperative learning: Prevalence, Conceptualisations, and the Relation Between Research and Practice. American Educational Research Journal, 35, (3), 419-454.

Aronson, E., Blaney, N ., Stephan, C., Sikes, J. \& Snapp, M . (1978). Thejigsaw dassroom. Beverly Hills, CA: Sage.

Baldwin, T.T. \& Ford, J.K. (1988). Transfer of training: a review and directions for future re search. Personnel Psychology, 41, 61-105. 
Bardin, L. (1977). L'analyse de contenu. Paris: PUF.

Blanchet, A. \& Gotman, A. (1992). L'enquête et ses méthodes: I'entretien. Paris: N athan.

Blaye, A. (1989). Interactions sociales et constructions cognitives: Présentation critique de la thèse du conflit socio-cognitif. In N . Bednarz \& C. G arnier (Ed.), Construction des savoirs. O bstacles et conflits (pp. 183-194). O ttawa: Cirade/Agence d'Arc.

Cauterman, M .-M ., D emailly, L., Suffys, S. \& Bliez-Sullerot, N . (1999). La formation continue des ensei gnants est-elle utile? Paris: Presses Universitai res de France.

Charlier, E. \& Charlier, B. (1998). La formation au cour de la pratique. Analyse d'une formation continuée d'enseignants. Paris-Bruxelles: D e Boeck \& Larcier.

Clark, C.M . \& Lampert, M . (1986). Q uel savoir sur l'enseignement pourrait être utile aux maitres? Q uel ques réflexions inspirées des recherches sur les aspects cognitifs des processus d'enseignement. In M . C rahay \& D . Lafontaine (Ed.), L'art et la science de l'enségnement (pp. 186-198). Bruxelles: Editions Labor.

Cohen, E. (1994, traduit par F. O uellet). Letravail de groupe: Stratégies d'enseignement pour la classe hétérogène. M ontréal: Editions L a C henelière.

Direction del'enseignement primaire. (2000). Les objectifs d'apprentissage del'écol e primaire genevoise. G enève: $D$ épartement de l'instruction publique.

Doise, W. \& M ugny, G. (1997). Psychologie sociale et développement cognitif. Paris: Armand Colin.

Gilly, M ., Fraisse, J. \& Roux, J.P. (1988). Résolution de problèmes en dyades et progrès cognitifs chez des enfants de 11 à 13 ans: dynamiques interactives et mécanismes socio-cognitifs. In A.-N . Perret-Clermont \& M . N icolet (Ed.), Interagir et connaître: Enjeux et ré gulations sociales dans le développement cognitif (pp. 73-92). Cousset (Fribourg): D elVal.

Gilly, M . (1988). Le fonctionnement de l'enfant à l'école. Journal européen de psychologie de l'éducation (numéro hors série). Lisbonne: ISPA.

H all, G.E. \& Loucks, S.F. (1977). A developmental model for determining whether the treatment is actually implemented. American Educational Research Journal, 14, 262-276.

H owden, J. \& Kopiec, M. (2000). Ajouter aux compétences. Enseigner, coopérer et apprendre au postsecondaire. M ontréal-Toronto: La Chenelière/M cG raw-H ill.

H uberman, M . (1989). La vie des enseignants, évolution et bilan d'une profession. Paris et $\mathrm{N}$ euchâtel: D elachaux et N iestlé.

Ishler, A.L., Johnson, R.T. \& Johnson, D.W. (1998). Long-term effectiveness of a statewide staff development program on cooperative learning. Teaching and Teacher Education, 14 (3), 273-281.

Johnson, D.W. \& Johnson, R.T. (1989). Cooperation and competition: Theory and Research. Edina: Interaction Book Company.

Johnson, D.W. \& Johnson, R.T. (1998). Effective staff development in cooperative learning: training, transfer, and long-term use. In C.M. Brody \& N. D avidson (Ed.), Professional de velopment for cooperative learning: Issues and approaches (pp. 223-242). N ew York: State University of N ew York Press.

Johnson, D.W., Johnson, R.T. \& Holubec, E. (1994). Cooperative learning in the classroom. Alexandria: ASCD.

Kagan, S. (1994). Cooperative learning. California: Resources for Teachers Inc.

Lehraus, K. (1998). Les enjeux de la coopération dans l'enseignement primaire. M émoire de licence en sciences de l'éducation, U niversité de G enève.

Perret-Clermont, A.-N . \& N icolet, M . (1988). (Ed.). Interagir et connaître: Enjeux et régulations sociales dans le dével oppement cognitif. Cousset (Fribourg): D elVal.

Raymond, D . (1993). Eclatement des savoirs et savoirs en rupture. U ne réplique à Van D er M aren. Revue des Sciences de l'Education, XIX (1), 187-200.

Rich, Y. (1990). I deological impediments to instructional innovation: T he case of cooperative learning. Teaching and Teacher Education, 6, 81-91. 
Roy, P. (1998). Staff development that makes a difference. In C.M. Brody \& N. Davidson (Ed.), Professional development for cooperative learning: Issues and approaches (pp. 79-99). $\mathrm{N}$ ew York: State U niversity of N ew York Press.

Sharan, S. (1994). (Ed.). H andbook of cooperativelearning methods. Westport, London: G reenwood Press.

Slavin, R.E. (1990). Cooperative Learning: Theory, Research and Practice. Englewood Cliffs: Prentice $\mathrm{H}$ all.

Smylie, M . (1988). The enhancement function of staff development: O rganizational and psychological antecedents to individual teacher change. American Educational Research Journal, 25, 1-30.

Sparks, G. (1988). Teacher attitudes toward change and subsequent improvements in classroom teaching. Journal of Educational Psychology, 80, 111-117.

Watson, M., Kenidzor, S., Dasho, S., Rutherford, S. \& Solomon, D. (1998). A social constructivist approach to cooperative learning and staff development: Ideas from the child development project. In C.M. Brody \& N . D avidson (Ed.), Professional development for cooperative learning: Issues and approaches (pp. 147-168). N ew York: State U niversity of N ew York Press.

\section{Kooperative Pädagogik: Von der Weiterbildung zur praktischen Umsetzung}

\section{Zus a mmenf assung}

D er vorliegende Artikel berichtet über eine Untersuchung zur praktischen U msetzung eines Ansatzes, auf den hin Genfer Primarlehrpersonen weitergebildet worden sind: der kooperativen Pädagogik. Verschiedene Probleme der Weiterbildung von Lehrpersonen werden ebenso dargestellt wie die methodologischen Aspekte der durchgeführten Untersuchung. D ie Datenanalyse zeigt, dass die überprüften Unterrichtsplanungen und die in den Interviews von den Lehrpersonen deklarierte U nterrichtspraxis allgemein mit den M erkmalen der kooperativen Pädagogik korrespondieren. Allerdings zeigen sich unterschiedliche Arten der U msetzung, was vor allem mit der Art und Weise zusammenhängt, wie den sozial en Aspekten des Ansatzes Rechnung getragen wird. D ieTeile der Resultate, welche die Faktoren betreffen, die die praktische U msetzung bestimmen, erlauben, einige Folgerungen für die kooperative Pädagogik in der Weiterbildung abzuleiten. 


\section{La pedagogia cooperativa: dalla formazione alla pratica}

Ri as sunt 0

Q uesto articolo èil resoconto di uno studio sulla concretizzazione di un metodo, la 'pedagogia cooperativa', a cui sono stati preparati degli insegnanti delle scuole elementari ginevrine. $\mathrm{Vi}$ sono presentate delle problematiche che concernono la formazione permanente degli insegnanti, come gli aspetti metodologici della stessa ricerca. L'analisi dei dati evidenzia il fatto che la pianificazione delle attività studiate e le pratiche descritte dagli insegnanti intervistati corrispondono in generale alle caratteristiche della pedagogia cooperativa. Tuttavia, il modo d'integrare l'aspetto sociale ha rilevato varie modalità di realizzazione. L'analisi dei fattori che ne influenzano la concretizzazione ha permesso di proporre alcuni suggerimenti utili per la formazione permanente alla pedagogia cooperativa.

\section{Cooperative pedagogy: from in-service training to practice}

\section{Summar y}

This article reports on a study focused on how primary school teachers in Geneva put into practice an instructional approach - cooperative pedagogy - for which they have received in-service training. Some issues concerning teacher inservice training are laid out, as well as methodological aspects of the present research. D ata analysis shows that the cooperative activities designed by the teachers and the practices declared by those interviewed correspond globally to the characteristics of cooperative pedagogy. H owever, various modes of implementation appear, linked especially to the way the social aspects of the approach are taken in account. The findings concerning the factors affecting the practice of this approach suggest some implications for in-service training in cooperative pedagogy. 